\title{
Monte Carlo-based Volumetric Arc Radiation Therapy vs. Helical Tomotherapy in Terms of Tumor Control Probability and Normal Tissue Complication Probability for Endometrial Cancers
}

\author{
(1) Sümeyra Can, (1) Illknur Harmankaya, (1) Özge Atilla, (1) Ayben Yentek Balkanay, \\ (1) Didem Karaçetin
}

University of Health Sciences Turkey, Başakşehir Çam and Sakura City Hospital, Clinic of Radiation Oncology, İstanbul, Turkey

\section{What is known on this subject?}

The dose range providing uncomplicated cure for gynecological cancers, especially in the presence of a gross disease, is narrow. Even though the provision of high-quality dose-response analysis for external radiotherapy of gynecologic carcinomas is not possible, analyses of tumor sites present an important correlation between the radiotherapy dose and probability of controlling macroscopic diseases. The treating doses used for lymph node metastases of gynecological cancers come with a limitation to reveal a significant relationship between dose and tumor response. A routine $60 \mathrm{~Gy}$ administration of radiotherapy to lymph node metastases with intensity modulated radiation therapy (IMRT) and image-guided radiation therapy leads to a significant decrease in the rate of intra-field paraaortic nodal recurrence to less than $5 \%$. These results offer a very significant relationship between the dose of radiotherapy and the tumor control probability (TCP). At the same time, the possibility of normal tissue complications for critical organs has gained importance in the evaluation of radiotherapy in recent years. For the same reason, the evaluation of normal tissue complication probability (NTCP) based on different methods for endometrial cancers has come to light in recent studies.

\section{What this study adds?}

The great importance of Monte Carlo (MC) dose calculation algorithm in protecting critical structures is determined in recent studies. Therefore, in this study, MC-volumetric arc radiation therapy (VMAT) plan was compared with the dose volume-helical tomotherapy (HT) plan to evaluate plan effectiveness in reducing the radiation dose causing toxicity and the quality of the plan was analyzed for both approaches in terms of dosimetric results, TCP and NTCP. For the analysis, two different approaches were considered for plan quality evaluation and the equivalent uniform dose (EUD) based TCP and NTCP model, proposed by Niemierko, was taken advantage of for analysis in this study. In previous studies, dosimetric analysis was done to evaluate critical structures' dose. However in the present study MC based VMAT plan HT plan in terms of EUD based TCP and norma tissue complication probability.

Address for Correspondence: Sümeyra Can MD, University of Health Sciences Turkey, Başakşehir Çam and Sakura City Hospital, Clinic of Radiation Oncology, İstanbul, Turkey Phone: +90 5536867040 E-mail: sumeyracn@gmail.com ORCID ID: orcid.org/0000-0003-1991-9474 Received: 26.01.2021 Accepted: 29.03.2021 


\section{ABSTRACT}

Objective: This study aimed to compare the effectiveness and to plan parameters of the Monte Carlo (MC)-based volumetric arc radiation therapy (VMAT) plan, which was devised using the equivalent uniform dose concept for endometrial cancers, to the dose volume (DV)-based helical tomotherapy (HT) plan. Additionally, both approaches were evaluated in terms of tumor control probability (TCP) and normal tissue complication probability (NTCP).

Material and Methods: The study comprised ten patients diagnosed with endometrial cancer, and treated with radixact tomotherapy unit. The target volumes (PTV) and organs at risks (OARs) were contoured through an accuracy planning system. All plans were devised to receive a total of $50.4 \mathrm{~Gy}$ in 28 fractions with the fractional dose to be $1.8 \mathrm{~Gy}$ for patient treatment. Monaco 5.51 planning system hosted all planning computed tomography images to devise MC-based VMAT plans. Both plans were analyzed in terms of TCP and NTCP.

Results: DV-HT plans (Cl: 1.1) came with the more conformal plan while the difference between both approaches was $<1 \%$ for $\mathrm{HI}$. Based on the results of the analyses, no statistical difference between DV-HT plan of MC-VMAT for the dose values of $2 \%, 30 \%$, and $40 \%$ of rectal volume $(p>0.05)$ was observed. The same results were obtained for the dose values of $2 \%$ and $30 \%$ of the bladder volume $(p>0.05)$. The $\mathrm{D}_{5 \%}$ of the femoral heads were $7 \mathrm{~Gy}$ which is $<$ MC-VMAT plan compared to DV-HT plan. The NTCP values of all OARs were $<1 \%$ in both approaches.

Conclusion: Statistically, similar results were obtained in MC-VMAT and DV-HT plans for OAR's doses when the treatment dose was given to PTV. Both approaches had no significant difference for NTCP statistically; however, the possibility of bone marrow complications to be investigated as well was concluded, so as to evaluate hematological toxicity.

Keywords: Endometrial cancer, Monte Carlo, NTCP, tomotherapy, TCP, VMAT

\section{Introduction}

Endometrial cancers (ECS) are among the most common forms of gynecological cancers worldwide (1). Predicted standard surgical treatment is by total abdominal hysterectomy (TAH) and bilateral salpingo-oopherectomy (2). Identification of lymph node-positive patients is recognized through lymphadenectomy compelling adjuvant therapy. However this therapy is not required in low-risk ECs (i.e., stage- 1 , grade I-II, $1 / 2<$ myometrial invasion, and no lymph vascular invasion) $(3,4,5)$. Based on the results of the GOG249 study, pelvic external beam radiation therapy (EBRT) should use as the standard therapy in patients with highintermediate and high-risk stage I-II EC (grade III, and deep invasion and/or lymph vascular space invasion, unfavorable histology, and unfavorable molecular factors) $(6,7)$. In the long run, EBRT increases the rise of morbidity; however, the pelvic region's acute and late toxicity is reduced by taking advantage of intensity modulated radiation therapy (IMRT) $(8,9,10)$. Additionally, a new dimension for IMRT is defined as the provision of highly conformal dose distribution within the target volume with helical tomotherapy (HT). Dose volume [(DV)-HT] planning is proven to be superior to a traditional linac-based IMRT in providing dose homogeneity and protecting the organs at risks (OARs) (11). One of the main advantages of IMRT compared to conformal radiation therapy (3D-CRT) is its ability to rapidly decrease provision in the dose between target volume (PTV) and OARs $(12,13,14)$. Nonetheless, controlling the low-dose region in the modern IMRT is proven to be difficult. Consequently, the risk of developing secondary malignancies in normal tissues is available. To avoid the problem, volumetric arc radiation therapy (VMAT) was developed; thus, the high dose area around the normal tissues was reduced at the same time by providing a homogeneous dose distribution in PTV. Meanwhile, controlling the low-dose zone with VMAT is easier $(15,16,17)$.

The Monaco treatment planning system (TPS) offers various optimizations for VMAT treatment (18). Unlike the DV-based TPS, Monaco TPS requires using three different biological functions for dose optimization, which are the poisson statistical cell kill model, serial, and parallel complication model $(19,20)$. Although the poisson statistical cell kill model is mandatory for target volumes, biological and physical function may be selected for OARs. In Monaco TPS, dose optimization takes place in two stages with beam segmentation performed in the first stage, as well as dose optimization in the second stage using the Monte Carlo (MC)based virtual source model $(21,22)$.

Undoubtedly, the foundation of radiotherapy is to provide maximum level of protection for the OARs, while delivering the prescription dose to the PTVs. On the same ground, being aware of the exact amount of absorbed dose plays an important role in escalating the chances of the success of the treatment, while protecting patients against radiation damage. Being aware of the tumor control probability (TCP) and normal tissue complication probability (NTCP), equivalent uniform dose (EUD) is the key to optimal plan design providing information about the treatment outcomes 
$(23,24,25)$. In recent years, the concept of EUD has gained importance in biological based treatment planning, since it reveals information about the organ function, whether serial or parallel $(26,27)$.

Considering the abovementioned concept, evaluating the effectiveness and plan parameters of MC-VMAT plan, which was created using the EUD concept, was aimed through comparing with DV-HT plan for ECs. Additionally, analyzing both approaches in terms of TCP and NTCP was aimed.

\section{Material and Methods}

\section{Patient Selection}

A total of ten patients diagnosed with ECs were selected for this retrospective study. All patients received adjuvant radiotherapy who were treated with Radixact Tomotherapy Unit in Basaksehir Cam, Sakura City Hospital Radiation Oncology Clinic between February 2021 and April 2021. All TAH, bisalpingo oophorectomy, and pelvic lymph node dissection were performed. Detailed information concerning the patients is presented in Table 1.

\section{Simulation and Contouring}

The planned computed tomography (CT) images were obtained by scanning the patients in the supine position

\begin{tabular}{|ll|}
\hline Table 1. Patient characteristics & \\
\hline Patient characteristics & Number of patients \\
\hline Myometrium invasion $<50 \%$ & 1 \\
\hline Myometrium invasion $>50 \%$ & 9 \\
\hline Grade I & 1 \\
\hline Grade II & 4 \\
\hline Grade III & 5 \\
\hline Endovascular invasion & 5 \\
\hline TAH + BSO + PLND & 7 \\
\hline TAH + BSO + PLND + PALND & 3 \\
\hline Peryton sampling (+) & 0 \\
\hline Peryton sampling (-) & 5 \\
\hline Peryton sampling (0) & 5 \\
\hline Stage IB & 6 \\
\hline Stage II & 2 \\
\hline Stage IIIA & 2 \\
\hline Adenocarcinoma & 9 \\
\hline Carcinosarcoma & 1 \\
\hline TAH: Total abdominal hysterectomy, BSO: & Bisalphingo-oophorectomy, \\
\hline PLND: Pelvic lymph mode dissection, PALND: Paraaortic lymph node \\
dissection & \\
\hline
\end{tabular}

with a slice of 3-mm thickness using a Philips Big Bore CT (Philips Healthcare, Andover, MA, USA). According to our defined protocol, all patients were asked to drink $1 \mathrm{~L}$ of water 45-60 minutes before the CT scan. At the same time, enemas were applied to the patients before the procedure, and the extraction was ensured with an empty rectum. All planned CT images were transferred with the Accuracy Precision of 2.0.0.1 TPS to contour the PTV and OARs. The radiation Oncology Group-0418 (RTOG) study atlas was used to control the nodal target volumes. Provided pelvic radiotherapy or common iliac, external, and internal iliac, obturator lymph nodes, parametrium, upper vaginal/paravaginal tissue, and presacral lymph nodes (in patients with cervical involvement) were observed, and they were included in the residual; in other ways, they were added in an operation lodge. A 1-cm wide vaginal volume was added laterally and caudally to the clinical target volume (CTV). A 7-mm margin was added to the periphery of the pelvic vessels, internal, external, and common iliac nodes. PTV was created by giving a 7-mm margin to the CTV. The bladder was contoured from the base to the dome. The rectum was contoured as the part between the ano-rectal line and the recto-sigmoid component. The peritoneal cavity was contoured up to $5 \mathrm{~cm}$ above the PTV. The femoral heads were contoured from the apex of the hip joint to the lower border of the lesser trochanter.

\section{Treatment Planning}

Taking advantage of Radixact Tomotherapy TPS, namely Accuracy Precision Version 2.0.0.1, DV-HT plans were devised (Tomotherapy Inc. Madison, WI). A total of 50.4 Gy to PTV in 28

Table 2. Summary of parameters used in all treatment plans

Energy $6 \mathrm{MV}$

Grid spacing $(\mathrm{cm})$

Algorithm

Pencil Beam and Monte Carlo

Statistical uncertainty $1 \%$ per calculation

Min. CT number $-600$

Auto flash margin $(\mathrm{cm})$

0.2

Surface margin $(\mathrm{cm})$

0.6

Beamlet width $(\mathrm{cm})$

0.3

Target margin Normal $(8 \mathrm{~mm})$

Avoidance margin Normal (8 mm)

Maximum number of arcs 2

Maximum control points $\quad 720$

Minimum segment width $(\mathrm{cm}) \quad 0.3$

Fluence smoothing Low 
fractions with the $1.8 \mathrm{~Gy}$ fractional dose was delivered during the treatment plans. The field width was determined as 2.5 $\mathrm{cm}$, pitch factor as 0.250 , and the modulation factor was selected as 3-3.5 in all plans. All contoured CT images were transferred to Monaco 5.51 TPs for the purpose of generating VMAT plans. Based on the biological optimization, EUD concept was used in MC-VMAT plans. The couch angle was $0^{\circ}$ and two arcs for a single arc with a fixed collimator rotational position at $0^{\circ}$ for all plans. The grid spacing, beamlet width, and minimum segment width were $0.3 \mathrm{~cm}$. In the first step, the pencil beam algorithm was used for rapid modeling, and the final dose optimization was done with the MC algorithm. The list of parameters used in all treatment plans is shown in Table 2. EUD-based functions for PTV and OARs were defined, and the list of functions used is presented in Table 3.

\section{Dosimetric Analysis}

Indices of conformity $(\mathrm{Cl})$ and heterogeneity $(\mathrm{HI})$ were used in this study to evaluate the plan quality. In addition, the $\mathrm{D}_{95 \%}$, $D_{98 \%}$, and $D_{2 \%}$ values which are the doses received by $95 \%$, $98 \%$, and $2 \%$ of PTV, respectively, and the mean dose $\left(D_{\text {mean }}\right)$ were analyzed. The volume receiving $107 \%$ of the treatment was considered to evaluate the maximum dose $\left(D_{\max }\right)$. The reference protocol for dose criteria of OARs was defined to

Table 3. The cost functions and isoconstraints that define the OARs and target

\begin{tabular}{|c|c|c|}
\hline \multicolumn{3}{|c|}{ MC-VMAT plan } \\
\hline Structure & Cost function & Isoconstraints \\
\hline \multirow[t]{2}{*}{ PTV } & Target penalty & PD: 5040 cGy \\
\hline & Quadratic overdose & $\begin{array}{l}\text { MD: } 5400 \text { cGy } \\
\text { RMS: } 2 \text { cGy }\end{array}$ \\
\hline \multirow[t]{2}{*}{ Bladder } & Parallel & $\begin{array}{l}\text { RD: } 3500 \text { cGy } \\
\text { MOD: } 40 \% \\
\text { PLE: } 3.5\end{array}$ \\
\hline & Serial & $\begin{array}{l}\text { EUD: } 3500 \text { cGy } \\
\text { PLE: } 15\end{array}$ \\
\hline \multirow[t]{2}{*}{ Rectum } & Parallel & $\begin{array}{l}\text { RD: } 2800 \text { cGy } \\
\text { MOD: } 20 \% \\
\text { PLE: } 3.5\end{array}$ \\
\hline & Serial & $\begin{array}{l}\text { EUD: } 2800 \\
\text { PLE: } 15\end{array}$ \\
\hline Femoral heads & Quadratic overdose & $\begin{array}{l}\text { MD: } 2000 \text { cGy } \\
\text { RMS: } 2 \text { cGy }\end{array}$ \\
\hline Bowel & Quadratic overdose & $\begin{array}{l}\text { MD: } 4000 \text { cGy } \\
\text { RMS: } 50 \text { cGy }\end{array}$ \\
\hline \multicolumn{3}{|c|}{$\begin{array}{l}\text { MD: Maximum dose, EUD: Equivalent uniform dose, RD: Reference dose, } \\
\text { PLE: Power low exponent, MOD: Mean organ damage, RMS: Root mean } \\
\text { square, MC: Monte Carlo, OARs: Organs at risks, VMAT: Volumetric arc } \\
\text { radiation therapy, PTV: Target volumes }\end{array}$} \\
\hline
\end{tabular}

be RTOG-0615 protocol. The $\mathrm{D}_{\text {max }}$ for the femoral heads, and the dose that received $5 \%$ of its volume $\left(\mathrm{V}_{50}\right)$ were taken into account. The dose received by $2 \%, 30 \%$, and $40 \%$ of the rectum and bladder volumes $\left(\mathrm{D}_{2 \%}, \mathrm{D}_{30 \%}, \mathrm{D}_{40 \%}\right)$, as well as the volume receiving $40 \mathrm{~Gy}\left(\mathrm{~V}_{40 \mathrm{~Gy}}\right)$ and $\mathrm{D}_{\text {mean }}$ were evaluated as well. Data from the DV histograms of all plans were used to determine the difference between the two approaches.

\section{Biological Model}

As Niemerko suggests, EUD-based TCP and NTCP were taken advantage of in radiobiological model response evaluation. To evaluate biological effectiveness, target dose distribution was performed based on a generalized EUD. The EUD was calculated according to the equation given below (28):

$$
g E U D=\left(\sum_{i} v_{i} D_{i}^{a}\right)^{1 / a}
$$

where $D_{i}$ is the dose, $v_{i}$, the fractional organ volume that received the dose, and $\mathrm{a}$ is the tissue-specific parameter that describes the DV effect (4).

In this study, $\mathrm{a}=-10$ was defined as the target volume. Additionally, biologically equivalent dose (EQD), which is the physical dose of $2 \mathrm{~Gy}$, was considered for the purpose of comparison. EQD was defined as

$$
E Q D=D \times \frac{\frac{\alpha}{\beta}+\frac{D}{n_{f}}}{\frac{\alpha}{\beta}+2}
$$

where $n_{f}$ is the fraction number, and $\alpha / \beta$ is linear quadratic parameter which is tissue-specific for organs (29). TCP, which is the probability of tumor cells controlling the radiation dose, was considered as well. TCP was calculated based on the equation

$$
T C P=\frac{1}{1+\left(\frac{T C D_{50}}{E U D}\right)_{50}} \cdots \cdots \cdots \cdots \cdots \cdots \cdots \cdots \cdots \cdots \cdots \cdots \cdots \cdots \cdots \cdots \cdots \cdots \cdots
$$

where $\mathrm{TCD}_{50}$ is the dose to control $50 \%$ of the tumor when the radiation is delivered to the tumor homogeneously. Based on the linear quadratic model, NTCP was defined as a function of the delivered dose and normal tissue volume which was irradiated. NTCP was calculated as

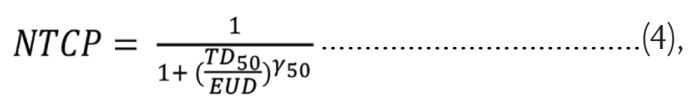

where $\mathrm{TD}_{50}$ is the tolerance dose for a $50 \%$ complication rate at a specific time interval, and $\gamma_{50}$ is a dimensionless parameter which defines the slope of the dose response curve (30). All coefficient used for EUD, EQD, TCP, and NTCP calculation are listed in Table 4. 


\begin{tabular}{|c|c|c|c|c|c|c|c|c|}
\hline Structure & $100 \%$ Dpf & $n_{f}$ & A & $\alpha / \beta(\mathrm{Gy})$ & $\gamma_{50}$ & $\mathrm{TCD}_{50}(\mathrm{~Gy})$ & $\mathrm{TD}_{50}(\mathrm{~Gy})$ & Dpf (Gy) \\
\hline Tumor & 1.8 & 28 & -10 & 1.2 & 2.2 & 28.34 & - & 2 \\
\hline Bladder & 1.8 & 28 & 2 & 8 & 2.66 & - & 80 & 2 \\
\hline Femur heads & 1.8 & 28 & 4 & 0.85 & 4 & - & 65 & 2 \\
\hline
\end{tabular}

\section{Statistical Analysis}

The dosimetric comparison occurred in two parts: Firstly, the radiation dose for PTV and ORAs were analyzed based on the aforementioned criteria. In the second part, both the approaches were evaluated through EQD, EUD, TCP, and NTCP comparisons. The statistical differences of each parameter obtained through all plans were examined by SPSS statistical software (SPSS, Statistics v22, Chicago, IL, USA). For statistical analysis, the test of the significance between two plan parameters was first applied to check whether the variables assume normality. Provided that the differences were distributed normally, paired-samples t-test were applied, or else, two related-samples test was applied. A $p$ value $<0.05$ was considered statistically significant for both tests.

\section{Results}

\section{Dosimetric Comparison for Target Volume}

To evaluate the superiority of each approach in terms of PTV coverage, the MC-VMAT plan and DV-HT plan were compared based on the abovementioned criteria. Based on the results, no statistical difference between DV-HT plan and MC-VMAT plan was observed in terms of $D_{98 \%}, D_{95 \%}, V_{107 \%}(p>0.05)$ along with the percentage difference between both approaches for the parameters was obtained lesser than $1.5 \%$. On the one side, the $D_{2 \%}$ value was $1.12 \%$ higher in MC-VMAT plan compared to DV-HT plan, which was statistically significant $(p<0.05)$. On the other side, both treatment approaches showed similar results in delivering prescription dose to the target as well as providing a target volume coverage based on the statistical analysis. $\mathrm{Cl}$ and $\mathrm{HI}$ values were considered to assess the plan quality. Even though a more conformal dose distribution was achieved by the DV-HT plan than expected (Cl: 1.1), the difference between $\mathrm{HI}$ values was $<1 \%$. The planning data of target volume are listed in Table 5.

\section{Dosimetric Comparison for OARs}

The required dose of OARs was gained through a comparison between the MC-VMAT plan and the DV-HT plan.
The two approaches revealed no statistical difference in the dose values of $\mathrm{D}_{2 \%}, \mathrm{D}_{30 \%}$, and $\mathrm{D}_{40 \%}$ which received $2 \%, 30 \%$, and $40 \%$ of the rectal volume and the $\mathrm{V}_{406 y}$ value, which was the volume receiving $40 \mathrm{~Gy}(\mathrm{p}>0.05)$. In the DV-HT plan, the $\mathrm{D}_{\text {mean }}$ of the rectum was approximately 4 Gy lower. For the bladder, the difference between both plans was $<1 \%$ for $D_{2}$ and $\mathrm{V}_{40 \mathrm{~Gy}}$ values. On the other hand, $\mathrm{D}_{\text {mean }}$ and $\mathrm{D}_{40 \%}$ were $3 \mathrm{~Gy}$ and 4 Gy higher, respectively, in the MC-VMAT plan compared to DV-HT plan. For the femoral heads, the $D_{5 \%}$ value in the MC-VMAT plan was 7 Gy lower than the DV-HT plan, and the MC-VMAT plan was more effective in reducing the femoral heads dose. In addition, $D_{\max }$ was approximately 6 Gy and 3 Gy less for the right and left femoral heads, respectively, in the MC-VMAT plan, and the difference between the approaches was statistically significant $(p>0.05)$. The critical organ doses obtained from both plans along with their comparisons are presented in Table 5. Additionally, half dose distributions of the MC-VMAT plan and DV-HT plan were shown in Figure 1.

\section{Biologic Model Evaluation}

With the aiming gaining an awareness of the response of target volume and normal tissues to radiation, EUD-based TCP and NTCP calculations were performed. The mean EQD and EUD in MC-VMAT plan were 1.73 Gy and 48.6 Gy, respectively, while these values were $1.76 \mathrm{~Gy}$ and $49.3 \mathrm{~Gy}$ in the DV-HT plan. No statistically significant difference between the EUD values for both approaches $(p>0.05)$ was observed. EUDbased TCP was calculated for PTV according to Niemierko model. Although TCP values in the MC-VMAT plan were $<1 \%$ compared to the DV-HT plan, this result caused a statistically significant difference $(p<0.05)$. In addition, NTCP calculation was performed for the rectum, bladder, and femoral heads. NTCP values were $<1 \%$ in both approaches and no statistical difference was observed between the values ( $p>0.05)$. EUD, EQD, TCP, and NTCP values calculated for both approaches are shown in Table 6. 


\begin{tabular}{|c|c|c|c|c|}
\hline & & MC-VMAT plan & DV-HT plan & $p(<0.05)$ \\
\hline \multirow{7}{*}{ PTV 50.4} & $D_{2 \%}(G y)$ & $53.19 \pm 0.25$ & $52.59 \pm 0.37$ & 0.016 \\
\hline & $D_{98 \%}(G y)$ & $48.10 \pm 0.31$ & $48.77 \pm 0.31$ & 0.050 \\
\hline & $D_{95 \%}(G y)$ & $49.27 \pm 0.15$ & $49.72 \pm 0.19$ & 0.050 \\
\hline & $\mathrm{D}_{\text {mean }}(\mathrm{Gy})$ & $51.27 \pm 0.16$ & $51.07 \pm 0.29$ & 0.022 \\
\hline & $\mathrm{V}_{107 \%}(\%)$ & $0.23 \pm 0.25$ & $0.10 \pm 0.07$ & 0.083 \\
\hline & $\mathrm{Cl}$ & $0.52 \pm 0.28$ & $1.11 \pm 0.08$ & 0.000 \\
\hline & $\mathrm{HI}$ & $1.07 \pm 0.00$ & $1.09 \pm 0.01$ & 0.050 \\
\hline \multirow{5}{*}{ Rectum } & $\mathrm{D}_{\text {mean }}(\mathrm{Gy})$ & $28.07 \pm 8.346$ & $24.98 \pm 6.39$ & 0.037 \\
\hline & $D_{2 \%}(G y)$ & $51.46 \pm 1.48$ & $51.62 \pm 1.65$ & 0.444 \\
\hline & $\mathrm{D}_{30 \%}(\mathrm{~Gy})$ & $36.06 \pm 12.11$ & $35.09 \pm 11.21$ & 0.203 \\
\hline & $\mathrm{D}_{40 \%}(\mathrm{~Gy})$ & $31.63 \pm 11.52$ & $28.59 \pm 10.61$ & 0.114 \\
\hline & $\mathrm{V}_{40 \mathrm{~Gy}}(\%)$ & $29.10 \pm 17.22$ & $26.68 \pm 14.03$ & 0.445 \\
\hline \multirow{5}{*}{ Bladder } & $\mathrm{D}_{\text {mean }}(\mathrm{Gy})$ & $34.40 \pm 8.76$ & $31.55 \pm 8.94$ & 0.022 \\
\hline & $D_{2 \%}(G y)$ & $52.23 \pm 0.66$ & $51.97 \pm 0.77$ & 0.139 \\
\hline & $\mathrm{D}_{30 \%}(\mathrm{~Gy})$ & $41.74 \pm 9.35$ & $40.47 \pm 10.06$ & 0.047 \\
\hline & $\mathrm{D}_{40 \%}(\mathrm{~Gy})$ & $38.25 \pm 0.11$ & $34.94 \pm 12.00$ & 0.017 \\
\hline & $\mathrm{V}_{40 \mathrm{~Gy}}(\%)$ & $43.48 \pm 27.29$ & $41.55 \pm 26.16$ & 0.169 \\
\hline \multirow{2}{*}{ Right femur } & $D_{5 \%}(G y)$ & $20.54 \pm 5.11$ & $27.76 \pm 6.53$ & 0.005 \\
\hline & $\mathrm{D}_{\max }(\mathrm{Gy})$ & $30.97 \pm 8.74$ & $36.10 \pm 8.98$ & 0.799 \\
\hline \multirow{2}{*}{ Left femur } & $D_{5 \%}(G y)$ & $21.15 \pm 4.28$ & $28.02 \pm 5.98$ & 0.007 \\
\hline & $\mathrm{D}_{\max }(\mathrm{Gy})$ & $33.94 \pm 7.82$ & $36.61 \pm 7.29$ & 0.095 \\
\hline
\end{tabular}

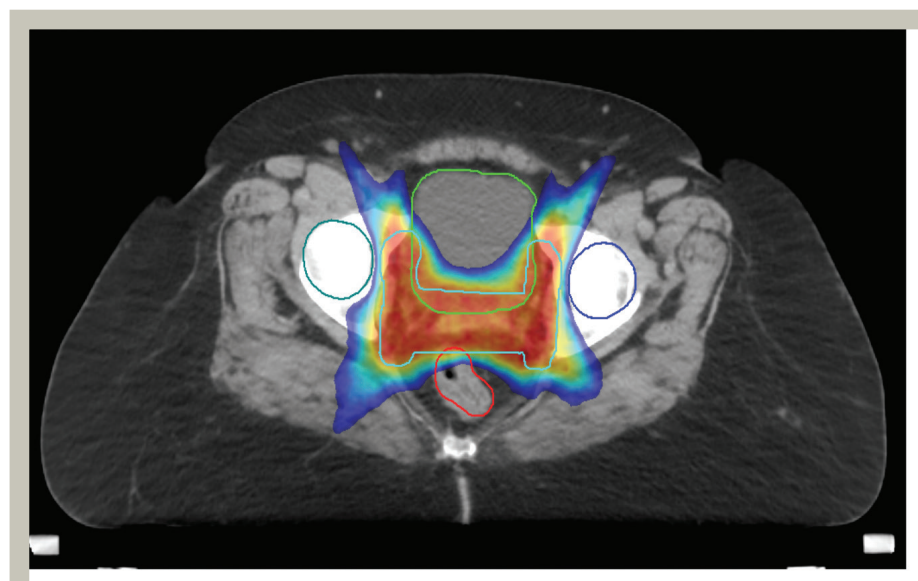

MC-VMAT plan

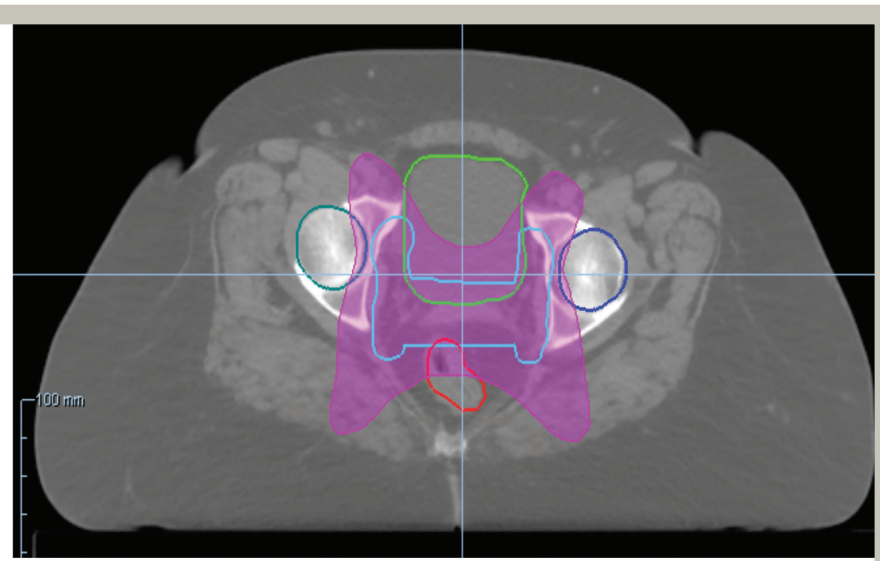

DV- HT plan

Figure 1. The half dose distribution in both approaches for the selected case

MC: Monte Carlo, VMAT: Volumetric arc radiation therapy, DV: Dose volume, HT: Helical tomotherapy 


\begin{tabular}{|c|c|c|c|}
\hline & MC-VMAT plan & DV-HT plan & $p(<0.05)$ \\
\hline $\mathrm{EQD}_{(\mathrm{PTV})}(\mathrm{Gy})$ & 1.735 & 1.760 & 0.008 \\
\hline $\mathrm{EUD}_{(\mathrm{PTV})}(\mathrm{Gy})$ & 48.60 & 49.30 & 0.277 \\
\hline TCP (\%) & 76.62 & 77.19 & 0.008 \\
\hline $\operatorname{NTCP}_{(\text {Rectum) }}(\%)$ & 0.065 & 0.047 & 0.107 \\
\hline $\operatorname{NTCP}_{\text {(Bladder) }}(\%)$ & 0.073 & 0.053 & 0.070 \\
\hline NTCP ${ }_{(\text {Right Femur Head) }}(\%)$ & 0.003 & 0.014 & 0.646 \\
\hline
\end{tabular}

\section{Discussion}

The dose range providing an uncomplicated cure for gynecological cancers, especially in the presence of a gross disease, is narrow. Even though the provision of highquality dose response analysis for external radiotherapy of gynecologic carcinomas is not possible, analyses of tumor sites present an important correlation between the radiotherapy dose and probability of controlling macroscopic diseases. The treating doses used for lymph node metastases of gynecological cancers come with a limitation to reveal a significant relationship between dose and tumor response. A routine 60 Gy administration of radiotherapy to lymph node metastases with IMRT and image-guided radiation therapy leads to a significant decrease in the rate of intrafield paraaortic nodal recurrence $<5 \%$. These results offer a very significant relationship between the dose of radiotherapy and the TCP. At the same time, the possibility of normal tissue complications for critical organs has gained importance in the evaluation of radiotherapy in recent years. For the same reason, the evaluation of NTCP based on different methods for ECs has come to light in recent studies.

Jodda et al. (31) compared NTCP values of bone marrow in ECs for different radiotherapy techniques and planning strategies. Data from 50 patients over three different treatment plans were analyzed. While evaluating the dose criteria for PTV, the rectum, bladder, bone marrow, bowel, and femoral heads, NTCP was compared for bone marrow only using the Lyman-Kuther-Burman-NTCP (LKB-TCP) model with the Bazan method (31).

Brent S. Rose et al. (25) tested whether the pelvic bone marrow radiation dose causes hematological toxicity in cervical patients, and the NTCP model was tried to be developed. In this study, the relationship between hematological subsets and $\mathrm{V}_{10 \mathrm{~Gy}}$ and $\mathrm{V}_{20 \mathrm{~Gy}}$ along with the volume of a bone marrow receiving 10 Gy and $20 \mathrm{~Gy}$, respectively, during chemoradiotherapy were analyzed. Based on the obtained results, hematological toxicity increased depending on the radiation dose received by the pelvic bone marrow volume (25).

Duman et al. (32) evaluated different treatment modalities, including 3D-CRT, field in field, and seven-field IMRT for patients with endometrial and cervical cancer. In their study, dosimetric comparisons were made for critical organs, and NTCP values were calculated for OARs. Additionally, they used LKB-NTCP models for the small intestine, rectum, and bladder; NTCP was $<1 \%$ for the rectum and bladder (32).

On the other hand, two different approaches were considered for plan quality evaluation and the EUD-based TCP and NTCP model proposed by Niemierko was taken advantage of for analysis in this study. The great importance of $M C$ dose calculation algorithm in protecting critical structures is determined in recent studies. Therefore, in this study, MC-VMAT plan was compared to the DV-HT plan to evaluate plan effectiveness in reducing the radiation dose causing toxicity, and the quality of the plan was analyzed for both approaches in terms of dosimetric results for TCP and NTCP. NTCP values of OARs were $<1 \%$ in both approaches, and there was no statistically significant difference between MC-VMAT and the DV-HT plan. However, this study does not consider bone marrow volume in the optimization process while hematological toxicity values were not included in the plan comparison.

\section{Conclusion}

This study compared the MC-VMAT plan to the DV-HT plan for EC. The plan parameters were analyzed in terms of TCP and NTCP. In the Monaco 5.51 TPS, VMAT plans were made using the MC algorithm and biologically based EUD concept. Similar TCP and NTCP values were obtained with MCVMAT plan as well as DV-HT plan. As a result of the analysis, both approaches achieved success in protecting OARs while 
delivering the prescription dose to PTV. On the other hand, the DV-HT plan was superior to the MC-VMAT plan in obtaining a more conformal dose distribution, and the MC-VMAT plan was superior to the DV-HT plan in reducing the $\mathrm{D}_{\max }$ and $\mathrm{D}_{5 \%}$ doses for the femoral heads. However, for a more detailed analysis, both approaches should be evaluated in terms of hematological toxicity.

\section{Ethics}

Ethics Committee Approval: Ethics Committee Approval is not required for dosimetric studies.

Informed Consent: Informed Consent form is not needed for dosimetric studies.
Peer-review: Internally peer-reviewed.

\section{Authorship Contributions}

Concept: S.C., Design: İ.H., A.Y.B., D.K., Data Collection or Processing: S.C., Ö.A., Analysis or Interpretation: S.C., I.H., D.K., Literature Search: S.C., Ö.A., I.H., Writing: S.C.

Conflict of Interest: No conflict of interest was declared by the authors.

Financial Disclosure: The authors declared that this study received no financial support.

\section{REFERENCES}

1. Amant F, Mirza MR, Koskas M, Creutzberg CL. Cancer of the corpus uteri. Int J Gynaecol Obstet 2015;131:S96-S104.

2. Creutzberg CL, Nout RA. The role of radiotherapy in endometrial cancer: current evidence and trends. Curr Oncol Rep 2011;13:472478.

3. Poulsen $\mathrm{H}$, Jacobsen $\mathrm{M}$, Bertelsen $\mathrm{K}$, et al. Adjuvant radiation therapy is not necessary in the management of endometrial carcinoma stage I, low-risk cases. Int J Gynecol Cancer 1996;6:38-43.

4. Lee CM, Szabo A, Shrieve DC, Macdonald OK, Gaffney DK. Frequency and effect of adjuvant radiation therapy among women with stage I endometrial adenocarcinoma. JAMA 2006 Jan;295:389-397. Erratum in: JAMA 2006;295:2482.

5. Naumann RW, Coleman RL. The use of adjuvant radiation therapy in early endometrial cancer by members of the Society of Gynecologic Oncologists in 2005. Gynecol Oncol 2007;105:7-12.

6. Harkenrider MM, Block AM, Alektiar KM, et al. American Brachytherapy Task Group Report: Adjuvant vaginal brachytherapy for early-stage endometrial cancer: a comprehensive review. Brachytherapy 2017;16:95-108.

7. Ao M, Ding T, Tang D, Xi M. Efficacy and toxicity of adjuvant therapies for high-risk endometrial cancer in stage I-III: a systematic review and network meta-analysis. Med Sci Monit 2020;26:e925595.

8. Lv Y, Wang F, Yang L, Sun G. Intensity-modulated whole pelvic radiotherapy provides effective dosimetric outcomes for cervical cancer treatment with lower toxicities. Cancer Radiother 2014;18:745-752.

9. Cozzi L, Dinshaw KA, Shrivastava SK, et al. A treatment planning study comparing volumetric arc modulation with RapidArc and fixed field IMRT for cervix uteri radiotherapy. Radiother Oncol 2008;89:180-191.

10. Nout RA, van de Poll-Franse LV, Lybeert ML, et al. Long-term outcome and quality of life of patients with endometrial carcinoma treated with or without pelvic radiotherapy in the post operative radiation therapy in endometrial carcinoma 1 (PORTEC-1) trial. J Clin Oncol 2011;29:1692-1700.
11. Marnitz S, Lukarski D, Köhler C, et al. Helical tomotherapy versus conventional intensity-modulated radiation therapy for primary chemoradiation in cervical cancer patients: an intraindividual comparison. Int J Radiat Oncol Biol Phys 2011;81:424-430.

12. Lian J, Mackenzie M, Joseph K, et al. Assessment of extendedfield radiotherapy for stage IIIC endometrial cancer using threedimensional conformal radiotherapy, intensity-modulated radiotherapy, and helical tomotherapy. Int J Radiat Oncol Biol Phys 2008;70:935-943.

13. Yang R, Xu S, Jiang W, Xie C, Wang J. Integral dose in three-dimensional conformal radiotherapy, intensity-modulated radiotherapy and helical tomotherapy. Clin Oncol (R Coll Radiol) 2009;21:706-712.

14. Hsieh CH, Shueng PW, Hsiao SM, et al. Helical tomotherapy provides efficacy similar to that of intensity-modulated radiation therapy with dosimetric benefits for endometrial carcinoma. Onco Targets Ther 2012;5:245-253.

15. Macchia G, Cilla S, Deodato F, et al. Simultaneous integrated boost volumetric modulated arc therapy in the postoperative treatment of high-risk to intermediate-risk endometrial cancer: results of ADA II phase 1-2 trial. Int J Radiat Oncol Biol Phys 2016;96:606-613.

16. Alongi F, Mazzola R, Ricchetti F, et al. Volumetric-modulated arc therapy with vaginal cuff simultaneous integrated boost as an alternative to brachytherapy in adjuvant irradiation for endometrial cancer: a prospective study. Anticancer Res 2015;35:2149-2155.

17. Yang R, Wang J, Xu F, Li H, Zhang X. Feasibility study of volumetric modulated arc therapy with constant dose rate for endometrial cancer. Med Dosim 2013;38:351-355.

18. Peters S, Schiefer H, Plasswilm L. A treatment planning study comparing Elekta VMAT and fixed field IMRT using the varian treatment planning system eclipse. Radiat Oncol 2014;9:153.

19. Clements M, Schupp N, Tattersall M, Brown A, Larson R. Monaco treatment planning system tools and optimization processes. Medical Dosimetry 2018;43:106-117.

20. Paudel MR, Kim A, Sarfehnia A, et al. Experimental evaluation of a GPU-based Monte Carlo dose calculation algorithm in the Monaco treatment planning system. J Appl Clin Med Phys 2016;17:230-241. 
21. Sarkar B, Manikandan A, Nandy M, Munshi A, Sayan P, Sujatha N. Influence of monte carlo variance with fluence smoothing in VMAT treatment planning with Monaco TPS. Indian J Cancer 2016;53:158161.

22. Wang Y, Chen L, Zhu F, Guo W, Zhang D, Sun W. A study of minimum segment width parameter on VMAT plan quality, delivery accuracy, and efficiency for cervical cancer using Monaco TPS. J Appl Clin Med Phys 2018;19:609-615.

23. Marks LB, Yorke ED, Jackson A, et al. Use of normal tissue complication probability models in the clinic. Int J Radiat Oncol Biol Phys 2010;76(Suppl 3):S10-19.

24. Palma G, Monti S, Conson M, Pacelli R, Cella L. Normal tissue complication probability (NTCP) models for modern radiation therapy. Semin Oncol 2019;46:210-218.

25. Rose BS, Aydogan B, Liang Y, et al. Normal tissue complication probability modeling of acute hematologic toxicity in cervical cancer patients treated with chemoradiotherapy. Int J Radiat Oncol Biol Phys 2011;79:800-807.

26. Choi B, Deasy J0. The generalized equivalent uniform dose function as a basis for intensity-modulated treatment planning. Phys Med Biol 2002;47:3579-3589.
27. Olafsson A, Jeraj R, Wright SJ. Optimization of intensity-modulated radiation therapy with biological objectives. Phys Med Biol 2005;50:5357-5379.

28. Niemierko A. Reporting and analyzing dose distributions: a concept of equivalent uniform dose. Med Phys 1997;24:103-110.

29. Rana S, Cheng C. Radiobiological impact of planning techniques for prostate cancer in terms of tumor control probability and normal tissue complication probability. Ann Med Health Sci Res 2014;4:167172.

30. Senthilkumar K, Maria Das KJ. Comparison of biological-based and dose volume-based intensity-modulated radiotherapy plans generated using the same treatment planning system. J Cancer Res Ther 2019;15(Supplement):S33-S38.

31. Jodda A, Urbański B, Piotrowski T, Malicki J. Relations between doses cumulated in bone marrow and dose delivery techniques during radiation therapy of cervical and endometrial cancer. Phys Med 2017;36:54-59.

32. Duman E, Inal A, Sengul A, Koca T, Cecen Y, Yavuz MN. Dosimetric comparison of different treatment planning techniques with International Commission on radiation units and measurements report-83 recommendations in adjuvant pelvic radiotherapy of gynecological malignancies. J Cancer Res Ther 2016;12:975-980. 